\title{
Insulin resistance contributes to multidrug resistance in HepG2 cells via activation of the PERK signaling pathway and upregulation of Bcl-2 and P-gp
}

\author{
XINYUE LIU ${ }^{1 *}$, LINJING LI $^{1 *}$, JING LI $^{1}$, YAN CHENG $^{2}$, JING CHEN $^{3}$, \\ MINGHUI SHEN ${ }^{1}$, SHANGDI ZHANG ${ }^{1}$ and HULAI WEI ${ }^{3}$ \\ ${ }^{1}$ Department of Clinical Laboratory Center, The Second Hospital of Lanzhou University; ${ }^{2}$ Experimental Center, \\ Northwest University for Nationalities; ${ }^{3}$ School of Basic Medical Sciences, \\ Lanzhou University, Lanzhou, Gansu 730000, P.R. China
}

Received October 31, 2015; Accepted January 11, 2016

DOI: $10.3892 /$ or.2016.4632

\begin{abstract}
Liver tumorigenesis frequently causes insulin resistance which may be used as an independent risk factor for evaluation of survival and post-surgery relapse of liver cancer patients. In the present study, HepG2/IR, an insulin resistant HepG2 cell line, was established by exposing HepG2 cells to $0.5 \mu \mathrm{mol} / 1$ of insulin for $72 \mathrm{~h}$, and comparison of HepG2/IR with the parental HepG2 cells indicated that the HepG2/IR cells showed significantly enhanced resistance to the most frequently used chemotherapeutics for solid tumors, such as cisplatin, 5-fluorouracil, vincristine and mitomycin. Flow cytometric analysis of cisplatin-treated HepG2/IR cells showed a significantly decreased hypodiploid peak and a significantly downregulated expression level of pro-apoptotic protein caspase- 3 compared with the parental HepG2 cells. Our data further showed swollen endoplasmic reticulum (ER) in the cisplatin-treated HepG2/IR cells with significantly increased levels of glucose-regulated protein 78 (GRP78), phosphorylated protein kinase R-like ER kinase (p-PERK) and P-glycoprotein (P-gp). There was also an upregulated expression of anti-apoptotic protein B-cell lymphoma 2 (Bcl-2) whereas no significant change was observed for CCAATenhancer-binding protein homologous protein (CHOP), which is known to be induced by ER stress and to mediate apoptosis. Our results demonstrated that insulin resistance in HepG2 cells promoted a protective unfolded protein response and upregulated the expression of ER chaperone protein GRP78, which resulted in the phosphorylation of PERK kinase to
\end{abstract}

Correspondence to: Dr Hulai Wei, School of Basic Medical Sciences, Lanzhou University, 199 Donggang West Road, Lanzhou, Gansu 730000, P.R. China

E-mail: hulaiweipaper@sina.com

${ }^{*}$ Contributed equally

Key words: insulin resistance, multidrug resistance, HepG2, PERK pathway, upregulation activate the PERK-mediated ER stress signal transduction pathway and the upregulation of Bcl-2 and P-gp, leading to the inhibition of the caspase-3-dependent apoptosis pathway and to the survival of liver tumor cells.

\section{Introduction}

Liver cancer is the most prevalent cancer in the clinic in China with a very high mortality rate of 26.04/100,000 cases. Treatment with conventional chemotherapeutics frequently produces unsatisfactory results mainly due to the acquisition of multidrug resistance (MDR) by the tumor cells. It has been suggested that MDR is caused by the upregulation of permeability $\mathrm{P}$-glycoprotein (P-gp), which leads to enhanced capability of cancer cells to pump chemotherapeutics out, thus preventing the intracellular accumulation of drugs. In addition, other factors, such as upregulation of anti-apoptotic proteins and abnormal apoptotic pathway are also involved in MDR (1). However, in recent years, a large number of epidemiological studies have revealed a close correlation between insulin resistance (IR) and liver cancer IR (2-4), a pathological condition in which insulin function is impaired in peripheral target tissues. This has been associated with various diseases including NIDDM, obesity, hypertension, dyslipidemia and atherosclerotic cardiovascular disease (5). Recent epidemiological and clinical evidence also links IR with cancer, including liver cancer $(6,7)$. As the target organ of insulin, a variety of pathological processes, such as tumorigenesis and inflammation, could lead to blockade in the insulin signaling pathway in liver cells and cause IR (8), which has been shown to be an independent risk factor affecting the survival and post-surgical relapse of cancer patients (9). However, the exact mechanisms of IR-induced tumor cell resistance to chemotherapeutics are still poorly understood.

Liver cells contain a large number of endoplasmic reticula (ER) for protein synthesis. It has been suggested that IR could trigger various cellular responses, such as enhanced oxidative stress and unfolded protein responses, and upregulation of glucose regulated protein 78 (GRP78), to control damage and restore normal ER functions and protein folding (10). 
Our previous study demonstrated that IR may lead to chemotherapeutic drug tolerance in liver cancer cells $(11,12)$, which could be caused by ER stress response (13). To further elucidate the mechanisms of IR-induced resistance to chemotherapeutics in liver tumor cells, the present study established an IR HepG2 cell line and investigated the possible involvement of the PERK signaling pathway. Our results demonstrated that IR can lead to liver cancer cell resistance to various chemotherapeutic drugs, including cisplatin. IR in HepG2 cells promoted the unfolded protein response; increased the expression of ER chaperone GRP78; phosphorylated PERK kinase to activate the PERK ER stress pathway; significantly upregulated the expression of B-cell lymphoma 2 (Bcl-2) and P-gp, all of which ultimately inhibited the caspase-3-dependent apoptosis pathway and promoted the survival of liver cancer cells. Notably, this entire process failed to significantly enhance the expression of CCAAT-enhancer-binding protein homologous protein (CHOP), indicating that IR only activated the unfolded protein response which is an early ER protective stress reaction.

Therefore, we hypothesized that IR promoted liver tumor cell tolerance to various chemotherapeutics through activation of the ER stress response PERK pathway and the upregulation of Bcl-2 and P-gp.

\section{Materials and methods}

Chemicals and antibodies. Insulin was purchased from Sigma-Aldrich (St. Louis, MO, USA) and diluted to the appropriate concentrations using $2 \%$ glacial acetic acid. The primers used to measure InsR, Glut- 2 and $\beta$-actin were synthesized by Takara Corporation (Japan). SYBR Premix Ex Taq and Prime Script RT reagents were also purchased from Takara. Cisplatin (DDP), 5-fluorouracil (5-FU), mitomycin C (MMC) and vincristine sulfate (VCR) were obtained from Sigma-Aldrich. The glucose content in the culture supernatant was measured with the GOD-POD method by Randox Laboratories (Crumlin, UK) using a Hitachi 7600 automatic biochemical analyzer (Hitachi, Tokyo, Japan).

Antibodies for insulin receptor, glucose transporter-2 and P-gp were purchased from Abcam (Cambridge, UK). Antibodies for caspase-3, Bcl-2, PERK and $\beta$-actin were purchased from Cell Signaling Technology (Boston, MA, USA). Antibodies for phosphorylated PERK and CHOP were obtained from Santa Cruz Biotechnology (Santa Cruz, CA, USA).

Cell culture and induction for IR. Human hepatocarcinoma HepG2 cells from the American Type Culture Collection (ATCC; Manassas, VA, USA) were maintained in Dulbecco's modified Eagle's medium (DMEM) supplemented with $10 \%$ fetal bovine serum (FBS) from HyClone (Logan, UT, USA) at $37^{\circ} \mathrm{C}$ with $5 \% \mathrm{CO}_{2}$. IR was induced in $\mathrm{HepG} 2$ cells according to a previously described method (12). Briefly, the cells were incubated in serum-free DMEM for $6 \mathrm{~h}$, and then treated with $0.5 \mu \mathrm{mol} / \mathrm{l}$ insulin for $72 \mathrm{~h}$. The resultant cells were named as HepG2/IR cells. HepG2/IR cells were then treated with PH $(10 \mathrm{mmol} / \mathrm{l})$ for $24 \mathrm{~h}$ to reverse IR, and the resultant cells were called HepG2/IR/PH cells.

Glucose oxidase-peroxide enzyme (GOD-POD) assay. HepG2 cells were stimulated with insulin as described above and cultured in DMEM without phenol red for $24 \mathrm{~h}$. Culture supernatants were then collected for the GOD-POD assay to measure glucose levels and calculate glucose consumption (12).

Periodic acid-Schiff (PAS) reaction assay. The PAS assay was used to assess glycogen synthesis (14). HepG2 and HepG2/IR cells were cultured on cover slides and fixed in $10 \%$ formaldehyde. Cells were then incubated with $1 \%$ periodate solution, washed, and then incubated in the dark with Schiff reagent for $20 \mathrm{~min}$ at $60^{\circ} \mathrm{C}$. The reactions were terminated when the color of the dye had changed from pink to purple-red. Optical density (OD) (as a measure of glycogen synthesis) was analyzed using an AX80 optical microscope (Olympus, Japan).

Ultrastructure. The morphological features were assessed using electron microscopy (15). Briefly, HepG2 and HepG2/IR cells were immobilized in $3 \%$ glutaraldehyde, embedded and sectioned. The ultrastructure was observed using a JEM 1230 transmission electron microscope (Jeol, Japan).

MTT assays. HepG2, HepG2/IR and HepG2/IR/PH cells were harvested and plated into 96-well plates at a density of $1 \times 10^{5}$ cells $/ \mathrm{ml}$, and then treated with $1.00-128.00 \mathrm{mg} / 1$ DDP, $12.50-1,600.0 \mathrm{mg} / 1$ 5-FU, $1.00-128.00 \mathrm{mg} / \mathrm{l} \mathrm{VCR}$ and $0.08-10.00 \mathrm{mg} / 1$ mitomycin C (MMC) (all from Sigma-Aldrich), respectively, for 44 and $68 \mathrm{~h}$. Drug cytotoxicity was then assessed using the MTT assay. OD was recorded at $490 \mathrm{~nm}$ using a PowerWave X plate reader (Bio-Tek, USA). Cell proliferation inhibition rates were calculated using the following formula: Cell proliferation inhibition rate $=[(\mathrm{OD}$ control - OD experiment)/OD experiment] x 100\%. The halfmaximal inhibitory concentration $\left(\mathrm{IC}_{50}\right)$ was also calculated as previously reported (12). MTT was also used in glucose consumption assays to calculate cell number thus that glucose consumption could be normalized to cell number.

Flow cytometric analysis (FCM). An Annexin V/propidium iodide (PI) double staining assay from Invitrogen (Carlsbad, CA, USA) was used to determine cell apoptosis. HepG2 and HepG2/IR cells were treated with DDP, 5-FU, VCR and MMC for $48 \mathrm{~h}$, then collected and suspended in binding buffer $(400 \mu \mathrm{l})$ and incubated with Annexin V-FITC and PI for $0.5 \mathrm{~h}$, and then suspended in binding buffer. The samples were analyzed by a Coulter Epics XL flow cytometer (Beckman Coulter, USA). The early apoptotic index was calculated as the percentage of Annexin V-positive and PI-negative cells. The later period index was assessed using the percentage of Annexin V-positive and PI-positive cells.

For cell cycle analysis, $1 \times 10^{6}$ cells were collected and fixed overnight in $70 \%$ ethanol at $4^{\circ} \mathrm{C}$. Cells were then washed with phosphate-buffered saline (PBS) and stained with PI for $30 \mathrm{~min}$ at room temperature in the dark. Cell cycle analysis was performed with a Coulter Epics XL flow cytometer (15).

Real-time quantitative RT-PCR. Total RNA was extracted from different cells using TRIzol reagent from Invitrogen. All primers used for the PCR amplification of $\beta$-actin, InsR and glut- 2 genes were designed and synthesized by Takara as shown in Table I. For qRT-PCR, 400 ng of RNA was used 
A

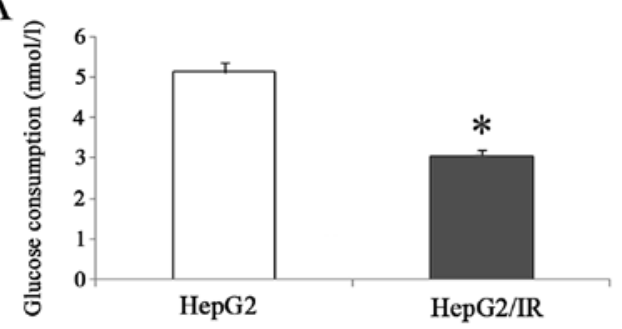

B

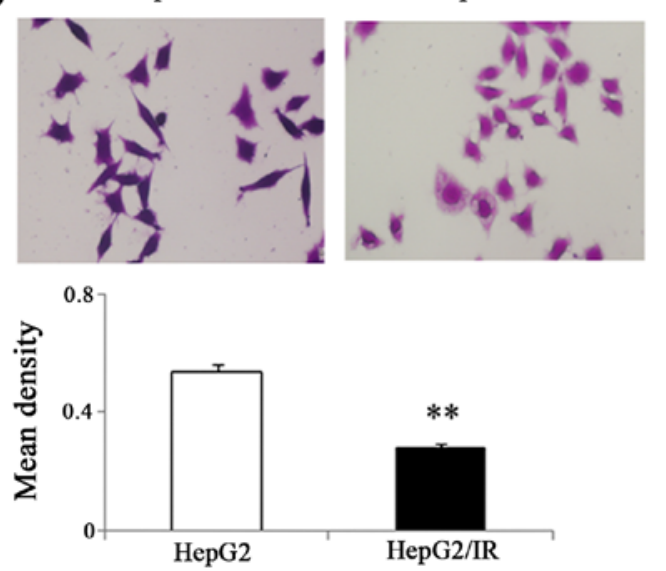

C

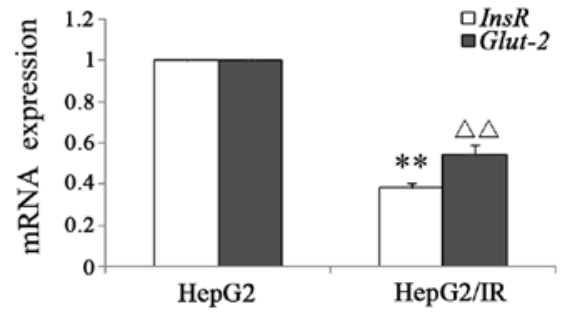

$\mathrm{D}$

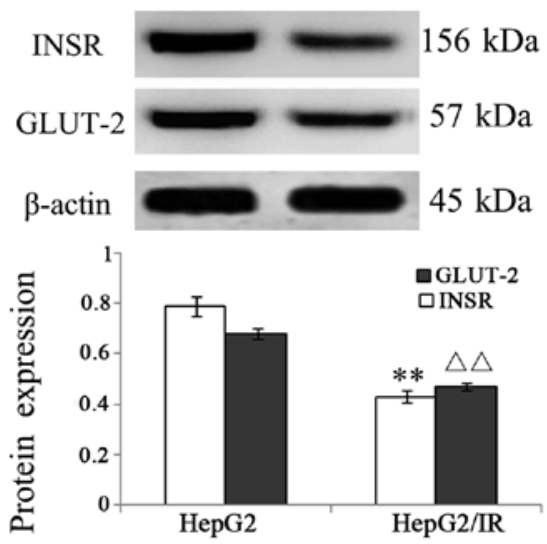

Figure 1. Analysis of insulin resistant HepG2/IR cells established by exposure of HepG2 cells to $0.5 \mu \mathrm{mol} / 1$ of insulin for $72 \mathrm{~h}$. (A) Glucose in the supernatants of HepG2/IR and HepG2 cells as measured by GOD-POD assay. (B) The glycogen content in the HepG2/IR and HepG2 cells was examined using PAS assay (upper panel) and photodensitometric analysis (lower panel). (C and D) The mRNA and protein levels of InsR and Glut-2 genes as determined by qRT-PCR and western blotting. All experiments were repeated three times. Data are presented as mean $\pm \mathrm{SD}$ of triplicate experiments. ${ }^{*}{ }^{*} \mathrm{P}<0.05 ;{ }^{* *, \Delta \Delta} \mathrm{P}<0.01$.

Table I. Primer sequences for qRT-PCR.

\begin{tabular}{lll}
\hline Gene & \multicolumn{2}{c}{ Primer sequence } \\
\hline InsR & 5' primer & 5'-TACCCTTCAAGAGATGATT-3' \\
& 3' primer & 5'-CAGAAGAAGTGGTGAAGAC-3' \\
Glut-2 & 5' primer & 5-TGGGCTGAGGAAGAGACTGT-3' \\
& 3' primer & 5'-AGAGACTGAAGGATGGCTCG-3' \\
$\beta$-actin & 5' primer & 5'-TGCTCCTCCTGAGCGCAAGTA-3' \\
& 3' primer & 5'-CCACATCTGCTGGAAGGTGGA-3' \\
& &
\end{tabular}

for cDNA synthesis with the PrimeScript ${ }^{\mathrm{TM}}$ RT Master Mix according to the manufacturer's protocol [Perfect Real-Time and qPCR was performed with the SYBR Premix Ex Taq (Tli RNase H Plus)] (both from Takara) using a Rotor-Gene 3000 quantitative PCR amplifier (Corbett, Australia). The PCR cycling conditions consisted of an initial denaturing phase at $95^{\circ} \mathrm{C}$ for $30 \mathrm{sec}$ followed by 35 cycles of $95^{\circ} \mathrm{C}$ for $5 \mathrm{sec}$ and $60^{\circ} \mathrm{C}$ for $30 \mathrm{sec}$. Data were analyzed with Rotor-Gene 6.0 software and relative gene expression was expressed as $2^{-\Delta \Delta \mathrm{Ct}}=(\mathrm{Ct}$ target gene - Ct housekeeping gene) $)_{\text {experimental group - }}$ ( Ct target gene - $\mathrm{Ct}$ housekeeping gene $)_{\text {control group }} \beta$-actin was used as the housekeeping gene for all the calculations.

Western blotting. Briefly, HepG2 cells under different treatments were lysed, and the protein concentrations were assessed using the Bradford assay from Roche (Basel,
Switzerland). Proteins were separated using SDS-PAGE electrophoresis and transferred to polyvinylidene difluoride (PVDF) membranes. The membranes were blocked with non-fat milk and probed with primary antibodies (anti-insulin receptor, anti-glut-2, anti-caspase3, anti-bcl-2, anti-GRP78, anti-PERK, anti-p-PERK, anti-P-gp or anti- $\beta$-actin) followed by IRDye $800 \mathrm{CW}$ or IRDye $700 \mathrm{DX}$-conjugated secondary antibodies from LI-COR Biosciences (Lincoln, NE, USA). Protein bands were visualized using an Odyssey double-color infrared laser imaging system (LI-COR). Relative expression was then calculated to reflect true protein expression (average infrared fluorescence intensity of the target protein $/ \beta$-actin).

Statistical analysis. Data are expressed as means \pm SD. Statistical analysis was performed using the Student's t-test with SPSS 15.0 (SPSS, Inc., Chicago, IL, USA). P-value $<0.05$ was considered to indicate a statistically significant result.

\section{Results}

IR in Hep G2/IR cells. IR was induced in HepG2 cells by incubation with $0.5 \mu \mathrm{mol} / 1$ insulin for $72 \mathrm{~h}$ to establish the HepG2/IR cell model. GOD-POD assay and PAS staining were used to measure the glucose consumption and glycogen synthesis. The relative glucose consumption in the HepG2/IR cells was significantly decreased when compared with the parental HepG2 cells $(\mathrm{P}<0.01$; Fig. 1A), and PAS staining revealed that the OD of intracellular glycogen in the HepG2/IR cells was decreased $45.10 \%$ compared with the HepG2 cells (Fig. 1B). 
A

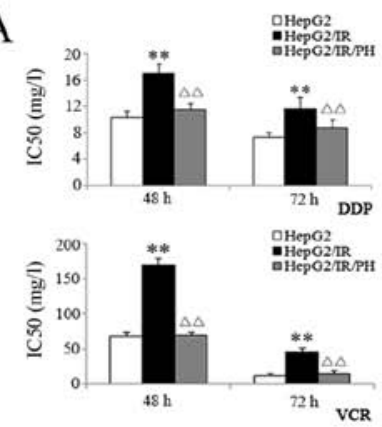

$\mathrm{C}$
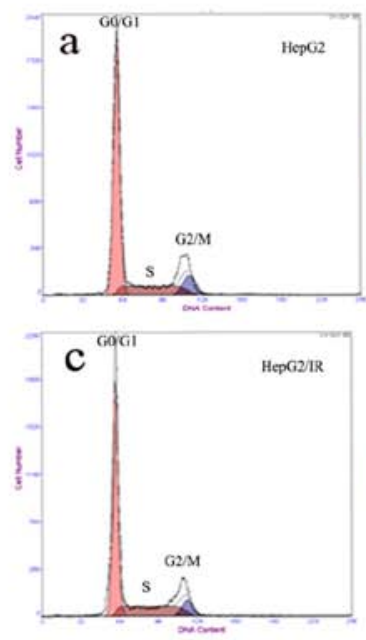

D
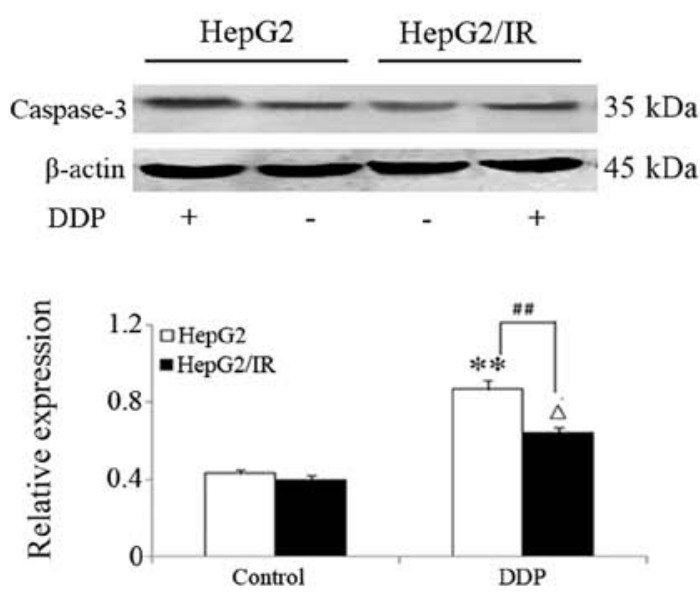
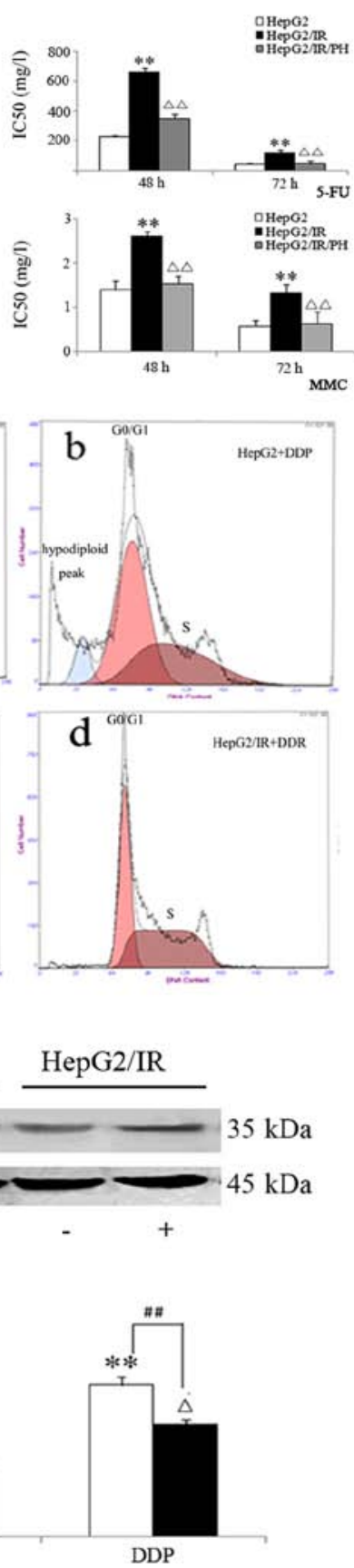
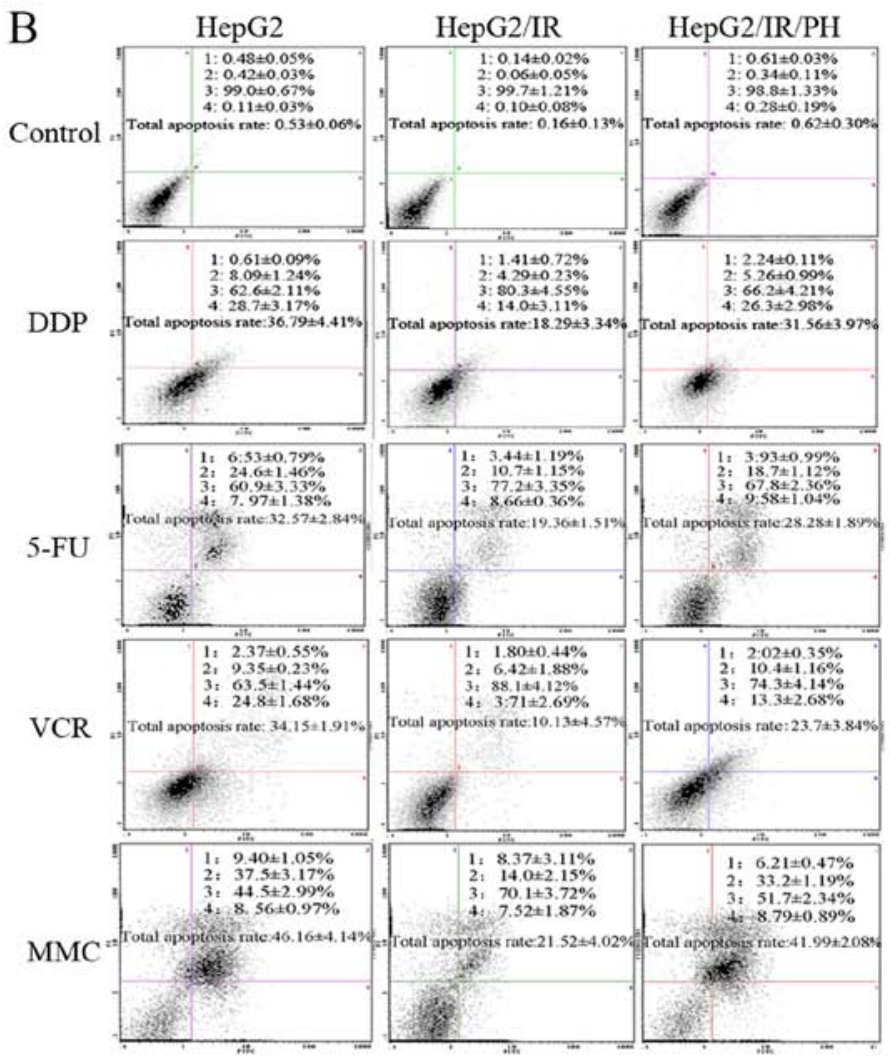

E
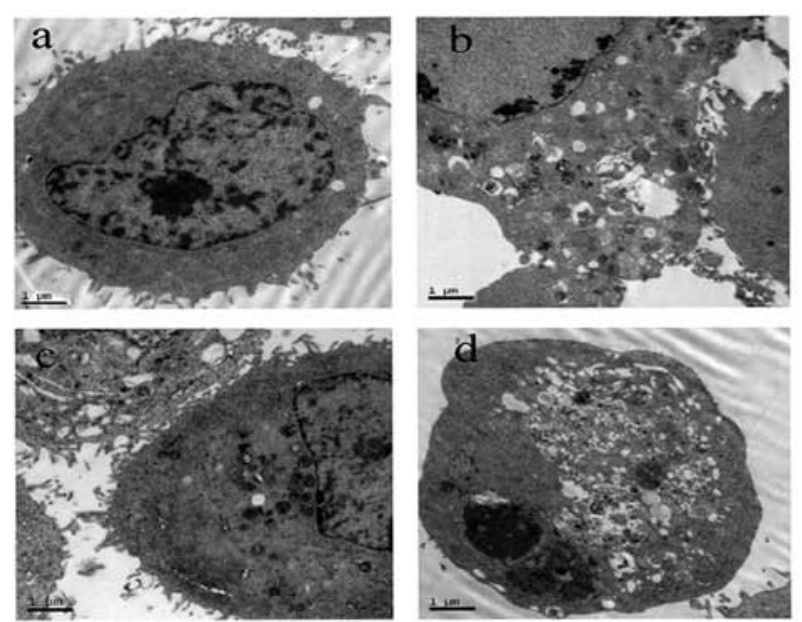

Figure 2. Analysis of multidrug resistance in the HepG2/IR cells. (A) Inhibition of proliferation in HepG2/IR, HepG2/IR/PH and HepG2 cells by various chemotherapeutic drugs at 48 and $72 \mathrm{~h}$ using the MTT assay. (B) Cell apoptosis at $48 \mathrm{~h}$ following treatment with different chemotherapeutic drugs using Annexin/PI double staining. (C) Cell cycle analysis of HepG2/IR and HepG2 cells at 48 h following DDP treatment. a, HepG2 cells; b, HepG2 cells treated with DDP for 48 h; c, HepG2/IR cells; and d, HepG2/IR cells treated with DDP for $48 \mathrm{~h}$. As indicated in the image, HepG2 cells treated with DDP showed a clear hypodiploid peak, which was absent from the HepG2/IR cells. (D) Western blot analysis of caspase-3 levels in the HepG2/IR and HepG2 cells before and after DDP treatment (upper panel). Lower panel shows the densitometric data from the western blotting. (E) Morphological changes associated with apoptosis in the HepG2/IR and the HepG2 cells after DDP treatment under electron microscopy (original magnification, x10,000): a, HepG2 cells; b, HepG2 cells treated with DDP; c, HepG2/IR cells; and d, HepG2/IR cells treated with DDP. All experiments were repeated three times, and data are presented as mean \pm SD of

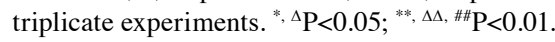

These results indicated that IR in HepG2/IR cells is associated with decreased glucose consumption and glycogen synthesis.

Results from the qRT-PCR analysis showed that the InsR mRNA level in HepG2/IR cells decreased to $62.00 \%$ and Glut-2 mRNA decreased to $58.23 \%$ of the levels in the parental HepG2 cells, respectively (Fig. 1C). Western blot analysis further confirmed the data from qRT-PCR with both INSR and GLUT-2 protein levels decreased (Fig. 1D).
MDR in HepG2/IR cells. HepG2/IR, HepG2/IR/PH and HepG2 cells were treated with DDP (1-64 mg/l), 5-FU (12.5-1,600 mg/l), VCR (1-128 mg/l) and MMC (0.08-10 mg/l) for 48 and $72 \mathrm{~h}$. MTT assay showed that cell proliferation was inhibited in all three groups, but HepG2/IR cells exhibited the least sensitivity towards the chemotherapeutics followed by HepG2/IR/PH and HepG2 cells. The $\mathrm{IC}_{50}$ values of HepG2/IR cells for DDP at 48 and $72 \mathrm{~h}$ were 1.66 and 1.59 times that 

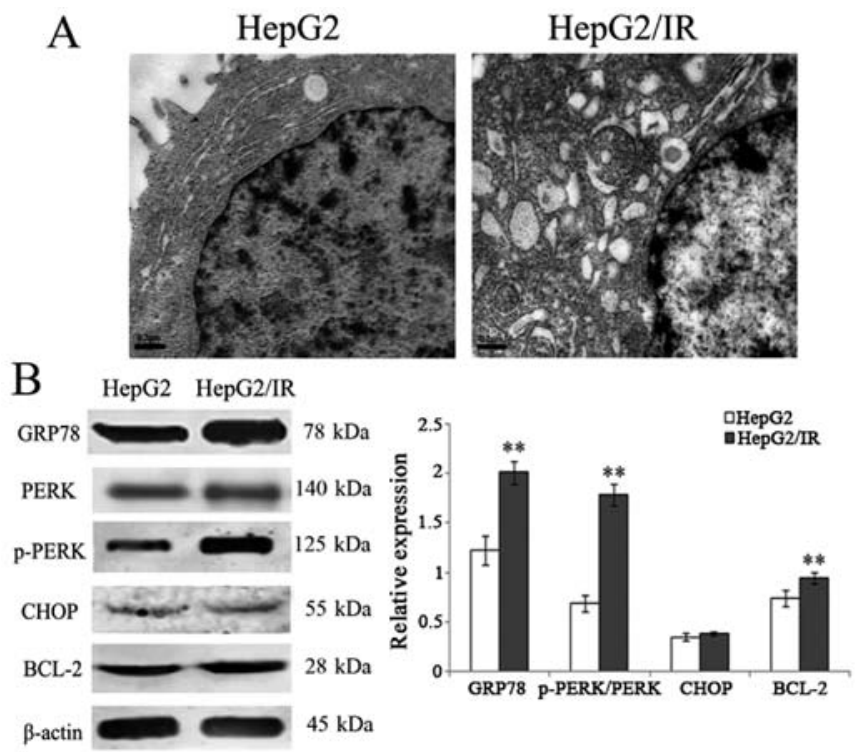

Figure 3. Activation of the ER PERK stress signaling pathway in HepG2/IR cells. (A) Morphological changes in the ER and mitochondria of HepG2/IR cells observed under electron microscopy. ER expansion and swollen mitochondria were obvious (original magnification, x20,000). (B) Western blotting (left panel) and densitometric analysis (right panel) of the expression levels of GRP78, PERK, p-PERK, CHOP and Bcl-2 in the HepG2/IR and HepG2 cells. All experiments were repeated three times and data are presented as mean $\pm \mathrm{SD}$ of triplicate experiments. ${ }^{* *} \mathrm{P}<0.01$.

of the parental HepG2 cells. However, after IR reversal with $10 \mathrm{mmol} / \mathrm{l} \mathrm{PH}$, the $\mathrm{IC}_{50}$ values of HepG2/IR/PH cells were 0.69 and 0.74 times that of the HepG2 cells, respectively. Similar trends, as shown in Fig. 2A, were also observed among the three different cell lines towards 5-FU, VCR and MMC. When cells were treated with different chemotherapeutics at concentrations of $\mathrm{IC}_{50}$ values for $48 \mathrm{~h}$, cell apoptosis was analyzed with flow cytometry using Annexin V/PI double staining. The results were consistent with data from the MTT assay (Fig. 2B). When HepG2 and HepG2/IR cells were treated with DDP at $16 \mathrm{mg} / \mathrm{l}$ for $48 \mathrm{~h}$, the hypodiploid peak was significantly reduced from $8.3 \%$ in $\mathrm{HepG} 2$ to $0.4 \%$ in the HepG2/IR cells (Fig. 2C). At the same time, western blot analysis showed that the expression of apoptotic protein caspase-3 decreased to $33.16 \%$ in the HepG2/IR cells compared to that parental levels in the HepG2 cells (Fig. 2D). Further analysis with TEM revealed cytoplasmic vacuolization and other morphological changes in the HepG2 cells associated with apoptosis. In the HepG2/IR cells, only a small number of vacuoles were observed (Fig. 2E).

Activation of the PERK signaling pathway in the HepG2/IR cells. To investigate ultrastructural changes in $\mathrm{HepG} 2$ cells after DDP treatment, cells were observed under an electron microscope. The results showed that the ultrastructure significantly changed in the HepG2/IR cells, such as ER expansion, ER degranulation, mitochondrial swelling, increased cell surface protrusions, and enlarged cell surface protrusions (Fig. 3A), indicating ER stress responses in the HepG2/IR cells.

To further elucidate the relationship between ER stress responses and apoptosis resistance in HepG2/IR, we further examined the proteins involved in the ER unfolded protein
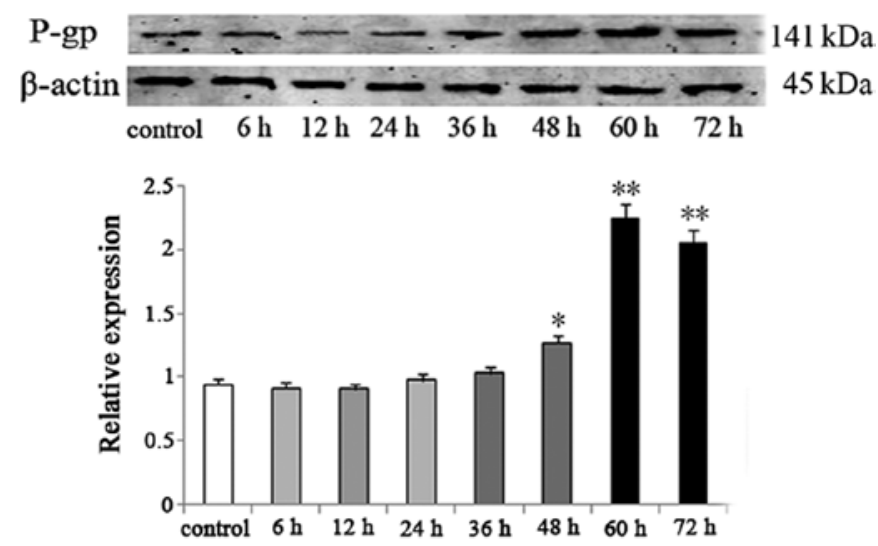

Figure 4. The expression of P-gp in the HepG2 cells during IR induction. P-gp protein levels at $6,12,24,36,48,60$ and $72 \mathrm{~h}$ after IR induction were analyzed by western blotting (upper panel), and the protein bands were analyzed by densitometry (lower panel). All experiments were repeated three times, and data are presented as mean $\pm \mathrm{SD}$ of triplicate experiments. ${ }^{* *} \mathrm{P}<0.01$.

response, including chaperone protein GRP78/Bip, PERK and phosphorylated-PERK which are important in the ER stress-related PERK pathway and CHOP which is important in the ER stress-related apoptosis pathway. In addition, we also observed a change in the expression of apoptosis resistance of the Bcl-2 protein. The results showed that the expression level of GRP78 increased $64.75 \%$ and the p-PERK/PERK ratio increased by 1.59 -fold in the HepG2/IR cells compared to the parental HepG2 cells. Notably, no significant change in CHOP expression was observed in the HepG2/IR cells. Furthermore, the expression of Bcl-2 also increased 27.03\% (Fig. 3B).

Upregulation of P-gp in the HepG2 cells during the induction of IR. P-gp has a drug reverse transport function, which can lead to high expression of the MDR gene in tumor cells. It is known that IR-induced hyperinsulinemia promotes the expression of P-gp, resulting in anticancer drug resistance in tumor cells (16). In addition, P-gp also plays protective roles as a chaperone for the transport of unfolded proteins during the ER stress reaction $(17,18)$. During the process of IR induction in the HepG2 cells, the expression of MDR protein P-gp was significantly upregulated. In comparison with the HepG2 cells, P-gp expression in the HepG2/IR cells increased $33.7 \%$ at $48 \mathrm{~h}$ $(\mathrm{P}<0.05)$ and continued to increase until reaching a peak at $>100 \%$ at $\sim 60 \mathrm{~h}(\mathrm{P}<0.01)$ (Fig. 4).

\section{Discussion}

Hepatocellular carcinoma (HCC) is the fifth most common malignancy and the third-leading cause of cancer-related death worldwide. Since HCC is often diagnosed at an advanced stage when it is no longer amenable to curative surgery, chemotherapy is the main treatment option in the clinic (19-21). However, the efficacy of first-line chemotherapeutics commonly used in the clinic for HCC treatment, such as doxorubicin and cisplatin, is not ideal (22). Thus, it has become imperative to elucidate the mechanisms underlying the resistance of HCC towards chemotherapeutics in order to find a more effective treatment strategy. 
Liver is an insulin sensitive organ and plays a critical role in the regulation of glucose metabolism and whole body energy homeostasis. The sensitivity of liver cells towards insulin can be reduced to create an IR condition when the insulin signaling pathway is blocked under pathological conditions (8). Mounting evidence suggests that IR is closely correlated with the initiation, progression and prognosis of various malignancies (2). It has been suggested that IR is a high risk factor for liver tumorigenesis and post-surgery relapse $(23,24)$. However, the role of IR in hepatocarcinoma has not been fully understood.

Recent studies suggest that the activation of the PI3K/Akt signaling pathway, the main pathway involved with IR, could cause tumor tolerance to various chemotherapeutic drugs (25). Alleviation of IR has been shown to increase patient sensitivity to chemotherapeutic drugs, reducing the progression of tumors and improving patient prognosis (26-28), indicating a possible correlation between IR and tumor tolerance to chemotherapeutics. In the present study, a comparison between high-dose insulin-induced IR HepG2/IR cells and parental HepG2 cells indicated that IR conferred tumor resistance to various chemotherapeutic drugs, such as cisplatin, 5-fluorouracil, vincristine and mitomycin. In addition, this resistance to chemotherapeutics was reversed by $\mathrm{PH}$, an insulin sensitizer. Our results suggested that IR decreased the sensitivity of HepG2 tumor cells towards chemotherapeutic drugs. Our results also showed that the hypodiploid peak and the expression of apoptotic protein caspase-3 in the HepG2/IR cells were significantly reduced after treatment with DDP, indicating that IR-induced inhibition of caspase-3-mediated cell apoptosis could be involved in the occurrence of resistance to chemotherapeutics. Previous studies suggest that IR triggers endoplasmic reticulum (ER) stress response (29-31). Imbalance in ER homeostasis could promote the accumulation of unfolded proteins to induce ER stress response, which is critical for cell survival. In contrast, chronic and severe ER stress response could also activate the apoptosis signal transduction pathway. Our ultrastructural data also showed signs of ER stress response in HepG2 cells after treatment with chemotherapeutics.

Emerging evidence suggests that ER stress is important in the drug resistance of tumor cells due to its involvement in cell survival. GRP78/Bip, an ER stress chaperone, was shown to help protein folding and transport during ER stress-induced unfolded protein response. External stress could induce a high expression of GRP78/Bip to maintain ER homeostasis and cell survival $(32,33)$. In the present study, the levels of GRP78/Bip, phosphorylated PERK and Bcl-2 in the HepG2/IR cells were significantly increased, which was consistent with previous observations. PERK, a member of the ER I transmembrane protein family, is a major player in the ER stress pathway. Under normal circumstances, PERK is in an inactive state by binding to GRP78. Accumulation of unfolded proteins can facilitate the dissociation of PERK from GRP78 which in turn causes the auto-phosphorylation of PERK. Phosphorylated PERK helps to block protein synthesis and to upregulate the expression of Bcl-2, leading to the survival of cells (34-36). In contrast, $\mathrm{CHOP} / \mathrm{GADD} 153$ could be upregulated to start the ER apoptosis pathway when the accumulated misfolded protein from long-term ER stress exceeds the capacity of the unfolded protein response (37). However, our data showed that no significant change in CHOP expression was observed between HepG2/IR and the parental HepG2 cells, suggesting that the development of IR only promoted the protective ER stress response and the activation of the PERK pathway was one of the mechanisms leading to the tolerance to chemotherapeutic drugs of the IR liver tumor cells.

P-gp, a drug transporter via ATP hydrolysis, has been linked with multidrug resistance (38). P-gp protein also functions as an ER chaperone protein to participate in the ER stress response by directly or indirectly transporting unfolded or misfolded protein and it could also provide protection for cells through inhibition of caspase-dependent cell apoptosis $(17,18)$. In the present study, the expression levels of the P-gp protein were studied in the HepG2 cells at 6, 12, 24, 48 and $72 \mathrm{~h}$ post high-dose insulin exposure. Our results showed that the expression level of P-gp was gradually increased during IR induction and peaked at $\sim 72 \mathrm{~h}$, indicating that $\mathrm{P}$-gp could be involved in the acquisition of drug tolerance in HepG2/IR cells.

In summary, our data demonstrated that multiple mechanisms are involved with the development of chemotherapeutic drug tolerance in HepG2/IR liver cancer cells, such as ER unfolded protein stress response, activation of the PERK signaling pathway, upregulation of $\mathrm{P}$-gp and anti-apoptotic protein Bcl-2, leading to the inhibition of the chemotherapeutic-induced caspase-3 pathway. However, further studies are needed to elucidate the full spectrum of the regulatory pathways of IR-mediated multidrug tolerance in cancer cells.

\section{Acknowledgements}

The present study was funded by The Project of the Youth Science and Technology Fund of Gansu Province of China (grant no. 1308RJYA055), the Scientific Research Initiation Funds for Doctor of the Second Hospital Affiliated to Lanzhou University, and the Program for Changjiang Scholars and Innovative Research Team in University (PCSIRT: IRT1137).

\section{References}

1. Huesker M, Folmer Y, Schneider M, Fulda C, Blum HE and Hafkemeyer P: Reversal of drug resistance of hepatocellular carcinoma cells by adenoviral delivery of anti-MDR 1 ribozymes. Hepatology 36: 874-884, 2002.

2. Nicolucci A: Epidemiological aspects of neoplasms in diabetes. Acta Diabetol 47: 87-95, 2010.

3. Donadon V, Balbi M, Perciaccante A, Casarin P and Zanette G: Insulin resistance and hyperinsulinemia in patients with chronic liver disease and hepatocellular carcinoma. Clin Med Insights Endocrinol Diabetes 2: 25-33, 2009.

4. Wang YG, Wang P, Wang B, Fu ZJ, Zhao WJ and Yan SL: Diabetes mellitus and poorer prognosis in hepatocellular carcinoma: A systematic review and meta-analysis. PLoS One 9: e95485, 2014.

5. DeFronzo RA and Ferrannini E: Insulin resistance. A multifaceted syndrome responsible for NIDDM, obesity, hypertension, dyslipidemia, and atherosclerotic cardiovascular disease. Diabetes Care 14: 173-194, 1991.

6. Tsugane $\mathrm{S}$ and Inoue $\mathrm{M}$ : Insulin resistance and cancer: Epidemiological evidence. Cancer Sci 101: 1073-1079, 2010.

7. Farrell G: Insulin resistance, obesity, and liver cancer. Clin Gastroenterol Hepatol 12: 117-119, 2014.

8. Leclercq IA, Da Silva Morais A, Schroyen B, Van Hul N and Geerts A: Insulin resistance in hepatocytes and sinusoidal liver cells: Mechanisms and consequences. J Hepatol 47: 142-156, 2007. 
9. Feng YH, Lin CY, Huang WT, Wu CL, Fang JL and Tsao CJ: Diabetes mellitus impairs the response to intra-arterial chemotherapy in hepatocellular carcinoma. Med Oncol 28: 1080-1088, 2011.

10. Quan X, Wang J, Liang C, Zheng $\mathrm{H}$ and Zhang L: Melatonin inhibits tunicamycin-induced endoplasmic reticulum stress and insulin resistance in skeletal muscle cells. Biochem Biophys Res Commun 463: 1102-1107, 2015.

11. Jong CJ, Ito T, Azuma $\mathbf{J}$ and Schaffer S: Taurine depletion decreases GRP78 expression and downregulates Perk-dependent activation of the unfolded protein response. Adv Exp Med Biol 803: 571-579, 2015.

12. Li LJ, Li GD, Wei HL, Chen J, Liu YM, Li F, Xie B, Wang B and Li CL: Insulin resistance reduces sensitivity to Cis-platinum and promotes adhesion, migration and invasion in HepG2 cells Asian Pac J Cancer Prev 15: 3123-3128, 2014.

13. Li L, Li G, Wei H, Sun J, Chen J, Xie B, Wang B, Gu J, Li C, Tian $\mathrm{B}$, et al: The endoplasmic reticulum stress response is associated with insulin resistance-mediated drug resistance in HepG2 cells. Neoplasma 62: 180-190, 2015.

14. Kurakula K, Hamers AA, van Loenen P and de Vries CJ: 6-Mercaptopurine reduces cytokine and Muc5ac expression involving inhibition of $\mathrm{NF} \kappa \mathrm{B}$ activation in airway epithelial cells. Respir Res 16: 73, 2015.

15. Li CL, Wei HL, Chen J, Wang B, Xie B, Fan LL and Li LJ: Arsenic trioxide induces autophagy and antitumor effects in Burkitt's lymphoma Raji cells. Oncol Rep 32: 1557-1563, 2014.

16. Zhou G and Kuo MT: NF-kappaB-mediated induction of mdrlb expression by insulin in rat hepatoma cells. J Biol Chem 272 : 15174-15183, 1997.

17. Ledoux S, Yang R, Friedlander G and Laouari D: Glucose depletion enhances P-glycoprotein expression in hepatoma cells: Role of endoplasmic reticulum stress response. Cancer Res 63: 7284-7290, 2003.

18. Morand JP, Macri J and Adeli K: Proteomic profiling of hepatic endoplasmic reticulum-associated proteins in an animal model of insulin resistance and metabolic dyslipidemia. J Biol Chem 280: 17626-17633, 2005.

19. Marrero JA, Kudo M and Bronowicki JP: The challenge of prognosis and staging for hepatocellular carcinoma. Oncologist 15 (Suppl 4): S23-S33, 2010.

20. Johnson PJ: Systemic chemotherapy of liver tumors. Semin Surg Oncol 19: 116-124, 2000

21. Roberts LR: Sorafenib in liver cancer - just the beginning. $N$ Engl J Med 359: 420-422, 2008.

22. Thorgeirsson SS and Grisham JW: Molecular pathogenesis of human hepatocellular carcinoma. Nat Genet 31: 339-346, 2002.

23. Nagaoki $Y$, Hyogo $H$, Aikata $H$, Tanaka M, Naeshiro N, Nakahara T, Honda Y, Miyaki D, Kawaoka T, Takaki S, et al: Recent trend of clinical features in patients with hepatocellular carcinoma. Hepatol Res 42: 368-375, 2012.

24. Huo TI, Wu JC, Lui WY, Lee PC, Huang YH, Chau GY, Tsay SH, Chang FY and Lee SD: Diabetes mellitus is a recurrence-independent risk factor in patients with hepatitis $B$ virus-related hepatocellular carcinoma undergoing resection. Eur J Gastroenterol Hepatol 15: 1203-1208, 2003.

25. Oki E, Baba H, Tokunaga E, Nakamura T, Ueda N, Futatsugi M, Mashino K, Yamamoto M, Ikebe M, Kakeji Y, et al: Akt phosphorylation associates with LOH of PTEN and leads to chemoresistance for gastric cancer. Int J Cancer 117: 376-380, 2005 .
26. Kourelis TV and Siegel RD: Metformin and cancer: New applications for an old drug. Med Oncol 29: 1314-1327, 2012.

27. Oliveras-Ferraros C, Cufí S, Vazquez-Martin A, Menendez OJ, Bosch-Barrera J, Martin-Castillo B, Joven J and Menendez JA: Metformin rescues cell surface major histocompatibility complex class I (MHC-I) deficiency caused by oncogenic transformation. Cell Cycle 11: 865-870, 2012

28. Shen Z, Ye Y, Bin L, Yin M, Yang X, Jiang K and Wang S: Metabolic syndrome is an important factor for the evolution of prognosis of colorectal cancer: Survival, recurrence, and liver metastasis. Am J Surg 200: 59-63, 2010

29. Yoshiuchi K, Kaneto H, Matsuoka TA, Kohno K, Iwawaki T, Nakatani Y, Yamasaki Y, Hori M and Matsuhisa M: Direct monitoring of in vivo ER stress during the development of insulin resistance with ER stress-activated indicator transgenic mice. Biochem Biophys Res Commun 366: 545-550, 2008.

30. Misra UK and Pizzo SV: Up-regulation of GRP78 and antiapoptotic signaling in murine peritoneal macrophages exposed to insulin. J Leukoc Biol 78: 187-194, 2005.

31. Kaneto H, Nakatani Y, Kawamori D, Miyatsuka T, Matsuoka TA, Matsuhisa M and Yamasaki Y: Role of oxidative stress, endoplasmic reticulum stress, and c-Jun N-terminal kinase in pancreatic beta-cell dysfunction and insulin resistance. Int $\mathrm{J}$ Biochem Cell Biol 38: 782-793, 2006.

32. Al-Rawashdeh FY, Scriven P, Cameron IC, Vergani PV and Wyld L: Unfolded protein response activation contributes to chemoresistance in hepatocellular carcinoma. Eur J Gastroenterol Hepatol 22: 1099-1105, 2010.

33. Lin JA, Fang SU, Su CL, Hsiao CJ, Chang CC, Lin YF and Cheng CW: Silencing glucose-regulated protein 78 induced renal cell carcinoma cell line G1 cell-cycle arrest and resistance to conventional chemotherapy. Urol Oncol 32: 29.e1-29.e11, 2014.

34. Nagelkerke A, Bussink J, van der Kogel AJ, Sweep FC and Span PN: The PERK/ATF4/LAMP3-arm of the unfolded protein response affects radioresistance by interfering with the DNA damage response. Radiother Oncol 108: 415-421, 2013.

35. Atkins C, Liu Q, Minthorn E, Zhang SY, Figueroa DJ, Moss K, Stanley TB, Sanders B, Goetz A, Gaul N, et al: Characterization of a novel PERK kinase inhibitor with antitumor and antiangiogenic activity. Cancer Res 73: 1993-2002, 2013.

36. Jiang HY and Wek RC: Phosphorylation of the $\alpha$-subunit of the eukaryotic initiation factor-2 (eIF $2 \alpha$ ) reduces protein synthesis and enhances apoptosis in response to proteasome inhibition. J Biol Chem 280: 14189-14202, 2005.

37. Feng J, Chen X, Sun X, Wang F and Sun X: Expression of endoplasmic reticulum stress markers GRP78 and CHOP induced by oxidative stress in blue light-mediated damage of A2E-containing retinal pigment epithelium cells. Ophthalmic Res 52: 224-233, 2014.

38. Gagliano T, Gentilin E, Benfini K, Di Pasquale C, Tassinari M, Falletta S, Feo C, Tagliati F, Uberti ED and Zatelli MC: Mitotane enhances doxorubicin cytotoxic activity by inhibiting P-gp in human adrenocortical carcinoma cells. Endocrine 47: 943-951, 2014. 